Mens

Revue d'histoire intellectuelle et culturelle

mens

\title{
Les origines intellectuelles de la commission \\ Laurendeau-Dunton : de la présence d'une volonté de dialogue entre les deux peuples fondateurs du Canada au lendemain de la Seconde Guerre mondiale, 1945-1965
}

Valérie Lapointe-Gagnon

Volume 14-15, numéro 2-1, printemps-automne 2014

La Commission royale d'enquête sur le bilinguisme et le biculturalisme a 50 ans : période révolue ou projet inachevé ?

URI : https://id.erudit.org/iderudit/1035529ar

DOI : https://doi.org/10.7202/1035529ar

Aller au sommaire du numéro

Éditeur(s)

Centre de recherche en civilisation canadienne-française

ISSN

1492-8647 (imprimé)

1927-9299 (numérique)

Découvrir la revue

Citer cet article

Lapointe-Gagnon, V. (2014). Les origines intellectuelles de la commission Laurendeau-Dunton : de la présence d'une volonté de dialogue entre les deux peuples fondateurs du Canada au lendemain de la Seconde Guerre mondiale, 1945-1965. Mens, 14-15(2-1), 131-173. https://doi.org/10.7202/1035529ar
Résumé de l'article

Cet article retrace les premières tentatives de dialogue et d'étude du bilinguisme et du biculturalisme au Canada qui ont précédé la commission Laurendeau-Dunton. À travers le récit de ces tentatives se dégage un portrait plus précis de l'histoire intellectuelle des années 1960, une décennie où les appels au dialogue et à la création d'un Canada respectant l'apport des deux peuples fondateurs se sont multipliés, culminant avec la mise sur pied de la commission Laurendeau-Dunton. Cette dernière rassemble un réseau d'intellectuels, déjà mobilisé autour des questions qu'elle aborde, soit la dualité, le bilinguisme et le biculturalisme, depuis les années qui ont suivi la Seconde Guerre mondiale. 


\section{Les origines intellectuelles de la commission Laurendeau-Dunton : de la présence d'une volonté de dialogue entre les deux peuples fondateurs du Canada au lendemain de la Seconde Guerre mondiale, 1945-1965}

Valérie Lapointe-Gagnon Campus Saint-Jean Université de l'Alberta

\section{Résumé}

Cet article retrace les premières tentatives de dialogue et d'étude du bilinguisme et du biculturalisme au Canada qui ont précédé la commission Laurendeau-Dunton. À travers le récit de ces tentatives se dégage un portrait plus précis de l'histoire intellectuelle des années 1960, une décennie où les appels au dialogue et à la création d'un Canada respectant l'apport des deux peuples fondateurs se sont multipliés, culminant avec la mise sur pied de la commission Laurendeau-Dunton. Cette dernière rassemble un réseau d'intellectuels, déjà mobilisé autour des questions qu'elle aborde, soit

\footnotetext{
${ }^{1}$ La rédaction de cet article a été rendue possible grâce au généreux soutien financier du Conseil de recherches en sciences humaines (CRSH) du Canada. Je remercie sincèrement les deux évaluateurs anonymes de cet article pour leurs commentaires pertinents et leurs suggestions judicieuses, qui ont contribué à peaufiner ma réflexion.
} 
la dualité, le bilinguisme et le biculturalisme, depuis les années qui ont suivi la Seconde Guerre mondiale.

\section{Abstract}

This article seeks to retrace the first attempts to study and practice bilingualism and biculturalism in Canada before the LaurendeauDunton Commission. The study of those attempts offers a clearer picture of the intellectual history of the 1960s, a decade that saw multiple calls to create a Canada more respectful of its two founding people, calls that culminated in the creation of the Laurendeau-Dunton Commission. This commission assembled a network of intellectuals that had already been mobilized in the preceding years on the questions that would occupy it: duality, bilingualism and biculturalism.

"Paris valait bien une messe : le Canada vaut peut-être une enquête ${ }^{2}$. " C'est ainsi que se conclut l'éditorial d'André Laurendeau de janvier 1962, qui a éveillé la conscience des responsables politiques et donné naissance à la Commission royale d'enquête sur le bilinguisme et le biculturalisme en juillet 1963. Si la plupart des articles et des chapitres d'ouvrages consacrés à la commission Laurendeau-Dunton s'entendent pour souligner le rôle déclencheur de l'éditorial de celui qui en devint le coprésident, il est également intéressant de rappeler que cet appel à une enquête approfondie avait lieu dans un contexte intellectuel plus large, marqué par un intérêt croissant des intellectuels canadiens et québécois pour les questions liées au bilinguisme et au biculturalisme depuis les années 1940 . Comme le mentionnait Jacques Julliard, les idées ne se promènent pas toutes nues dans la rue $\mathrm{e}^{3}$. Il faut des gens pour les porter et des contextes propices à les accueillir. C'est au climat intellectuel

2 André Laurendeau, "Pour une enquête sur le bilinguisme ", Le Devoir, 20 janvier 1962 , p. 4.

3 Jacques Julliard, "Le fascisme en France ", Annales, Économies, Sociétés, Civilisations (A.E.S.C.), 39e année, $\mathrm{n}^{\circ} 4$ (juillet-août 1984), p. 855. 
précédant la Commission, un climat empreint d'inquiétudes face à l'avenir du pays, que s'attachera cet article afin de montrer dans quel terreau intellectuel s'inscrit cette vaste entreprise de consultation publique et de recherches exhaustives sur le passé, le présent et l'avenir du Canada.

Dans quel contexte Lester B. Pearson a-t-il mis sur pied la Commission royale d'enquête sur le bilinguisme et le biculturalisme? Quels étaient les thématiques et les enjeux préoccupant les cercles intellectuels et politiques dans les années précédant la Commission? Cet article souhaite répondre à ces questions en retraçant les nombreuses tentatives effectuées à partir des années 1940 pour rapprocher le Québec et le reste du Canada. La création d'un terrain propice à la réforme des relations entre les deux peuples fondateurs constituait d'ailleurs un des objectifs ultimes de la commission LaurendeauDunton. Peu d'études ont abordé ces efforts de dialogue et de réconciliation. Certes, il y a l'article de Robert Talbot consacré à la Ligue de la Bonne Entente, qui met en lumière la nature de ce mouvement né pendant la Première Guerre mondiale et les motivations de ses acteurs, désireux de sauver le pays d'un éventuel morcèlement ${ }^{4}$. Toutefois, cet article constitue plutôt l'exception que la règle ${ }^{5}$. Robert

${ }^{4}$ Robert Talbot, «Une réconciliation insaisissable : le mouvement de la bonne entente, 1916-1930 ", Mens : revue d'histoire intellectuelle de l'Amérique française, vol. 8, nº 1 (automne 2007), p. 67-125, [En ligne], [http://id.erudit.org/iderudit/1023148ar].

5 D'autres ouvrages ont également porté sur les rapprochements entre anglophones et francophones, notamment les suivants : Patrice A. Dutil, «Against Isolationism: Napoléon Belcourt, French Canada, and "La grande guerre" ", dans Robert Craig Brown et David Mackenzie (dir.), Canada and the First World War: Essays in Honour of Robert Craig Brown, Toronto, University of Toronto Press, 2005, p. 96-137; et Robert Choquette, Language and Religion: A History of English-French Conflict in Ontario, Ottawa, Les Presses de l'Université d'Ottawa, 1975. Dans son ouvrage consacré à la crise de la conscription, André Laurendeau revient lui-même sur sa tentative de créer des liens avec un cercle d'universitaires de McGill, autour duquel gravitaient Frank Scott et Neil Morrison, pour lutter contre la conscription. Cet effort conjoint ne mena toutefois nulle part et laissa un goût amer dans la bouche de l'intellectuel québécois. Les anglophones abandonnèrent l'idée de conjuger leurs efforts à ceux des francophones lorsqu'éclata la guerre. Laurendeau écrit alors : "J'ai éprouvé ce jour-là une indignation et une amertume difficiles à surmonter : 
Talbot souligne d'ailleurs un fait intéressant des historiographies canadienne et québécoise, soit que les éléments de division entre anglophones et francophones ont fait couler beaucoup plus d'encre que les tentatives de réconciliation ${ }^{6}$. Son article met non seulement en lumière la profondeur des racines historiques de la volonté de rapprochement - même si certains se sont interrogés sur les véritables motifs des bon-ententistes -, mais aussi l'angoisse constante partagée par des intellectuels depuis la création du pays de le voir se désagréger. Pour notre part, nous allons nous consacrer à la question des rapprochements entre les anglophones et les francophones au cours de la période qui a suivi la Seconde Guerre mondiale puisque c'est à ce moment que les départements de sciences sociales se développent et qu'émerge une volonté chez les universitaires de comprendre et de documenter la dualité culturelle au pays. C'est aussi pendant ces années que le Canada traverse une période de questionnement identitaire qui pousse les intellectuels à s'interroger sur la nature même du caractère canadien, sur ce qui distingue le Canada et en fait un pays à part entière. Cet article veut donc reconstituer le climat intellectuel de l'époque, un climat qui révèle que la Commission n'a pas surgi de nulle part et qu'elle s'inscrit dans une longue série d'efforts de dialogue plus ou moins fructueux qui ont ponctué les années 1940, 1950 et 1960. Ce contexte restitué, un pan de l'histoire intellectuelle du Canada émerge de l'ombre : celui où des hommes et des femmes de différents horizons ont tenté de créer un dialogue porteur de solutions et de penser la dualité canadienne.

malgré de longs efforts, les ponts que nous avions voulu jeter d'une nation à l'autre étaient emportés, comme des fétus " (André Laurendeau, La crise de la conscription, 1942, Montréal, Éditions du Jour, 1962, p. 27). Ce qui ressort surtout de cette littérature consacrée à la réconciliation, c'est que, même si les contacts entre anglophones et francophones ont été plus nombreux que l'on pourrait croire, le fruit n’était pas tout à fait mûr au cours des années qui ont précédé la commission Laurendeau-Dunton.

6 Talbot, «Une réconciliation insaisissable », p. 69. 
Cet article veut également contribuer à enrichir notre compréhension de la commission Laurendeau-Dunton, dont plusieurs aspects sont demeurés occultés. La plupart des études qui ont porté sur cette commission ont été rédigées par ceux qui y ont participé Elles $^{2}$ permettent donc de jeter un regard inédit sur ce qui s'est passé entre les murs de cette aventure politique; toutefois, elles sont teintées du jugement et des opinions de leurs auteurs, qui mettent en valeur leur conception de la Commission. Il y a également eu des études pertinentes et exhaustives consacrées à l'héritage linguistique de cette commission créée pour penser la nation canadienne, notamment celles de Matthew Hayday ${ }^{8}$ et de Daniel Bourgeois9. Certaines publications ont été consacrées à des personnalités phares de cette commission. Ainsi, les rôles joués par André Laurendeau ${ }^{10}$ et Frank

7 Voir, notamment, Léon Dion, La révolution déroutée, 1960-1976, Montréal, Éditions du Boréal, 1998. Voir également l'article de Michael Oliver, «Laurendeau et Trudeau : leurs opinions sur le Canada ", dans Raymond Hudon et Réjean Pelletier (dir.), L'engagement intellectuel : mélanges en l'honneur de Léon Dion, Sainte-Foy, Les Presses de l'Université Laval, 1991, p. 339-368. Le commissaire Jean-Louis Gagnon parle abondamment de son parcours à la Commission dans ses mémoires : Les apostasies, t. 1-3, Montréal, La Presse, 1985. Le journal d'André Laurendeau, paru chez VLB éditeur, permet, quant à lui, de parcourir les travaux de la Commission à travers la vision de son coprésident (André Laurendeau, Journal tenu pendant la Commission royale d'enquête sur le bilinguisme et le biculturalisme, Montréal, VLB éditeur, 1990).

8 Matthew Hayday, Bilingual Today, United Tomorrow: Official Languages in Education and Canadian Federalism, Montréal, McGill-Queen's University Press, 2005, p. 44. Le chapitre consacré aux travaux de la Commission s'intitule «From Royal Commission to Government Policy ", p. 35-62.

9 Pour une histoire et une critique du sort réservé à la recommandation des commissaires de créer des districts bilingues, voir Daniel Bourgeois, The Canadian Bilingual Districts: From Cornerstone to Tombstone, Montréal, McGill-Queen's University Press, 2006.

${ }^{10}$ Voir le collectif dirigé par Robert Comeau et Lucille Beaudry, André Laurendeau, un intellectuel d'ici, Québec, Presses de l'Université du Québec, 1990; Denis Monière, André Laurendeau et le destin d'un peuple, Montréal, Québec Amérique, 1983; et le collectif dirigé par Nadine Pirotte, Penser l'éducation: nouveaux dialogues avec André Laurendeau, Montréal, Éditions du Boréal, 1989. Sur la formation de la pensée d'André Laurendeau, plus particulièrement sur sa conception du nationalisme, voir Yvan Lamonde, «André Laurendeau en Europe (1935-1937) : 
Scott ${ }^{11}$ sont bien détaillés, et la confrontation entre les deux hommes au sujet de leur conception respective du Canada est documentée ${ }^{12}$. Il n'en reste pas moins que certaines questions demeurent, notamment en ce qui a trait au contexte de la mise sur pied de cette commission, souvent rapidement effleuré, mais essentiel puisqu'il éclaire la richesse de son héritage intellectuel ${ }^{13}$.

Notre étude se scindera en trois parties. La première portera sur le contexte politique des années de l'après-guerre, un contexte où les réflexions sur l'avenir du Canada se multiplient alors que le pays, nouvellement indépendant, aspire à trouver sa voie sur les scènes nationale et internationale. La deuxième partie sera consacrée à la

la recherche d'un nouvel ordre ", Les Cahiers des Dix, nº 61 (2007), p. 215-251. Sur la participation de Laurendeau à la Ligue d'action nationale, voir Pascale Ryan, Penser la nation : la Ligue d'action nationale, 1917-1960, Montréal, Leméac éditeur, 2006. Sur sa participation à la commission Laurendeau-Dunton, voir Richard Arès, "André Laurendeau et la Commission B.B. ", Relations, $\mathrm{n}^{\circ} 329$ (juillet-août 1968), p. 210-212, et l'introduction de Paul Lacoste dans Laurendeau, Journal tenu pendant la Commission royale d'enquête sur le bilinguisme et le biculturalisme. Ce qui ressort des ouvrages et articles écrits sur le parcours d'André Laurendeau, c'est que les premières années de son engagement politique sont beaucoup mieux documentées que les dernières années de sa vie et sa contribution à la commission Laurendeau-Dunton.

11 Sur Frank Scott, voir Sandra Djwa, "Nothing by Halves" : F. R. Scott ", Journal of Canadian Studies, vol. 34, no 4 (hiver 1999-2000), p. 52-69; Sandra Djwa, F. R. Scott : une vie, traduit de l'anglais par Florence Bernard, Montréal, Éditions du Boréal, [1987] 2001 ; Allen Mills, "Of Charters and Justice: The Social Thought of F. R. Scott, 1930-1985 ", Journal of Canadian Studies, vol. 32, n 1 (printemps 1997), p. 44-62. Susan Margaret Murphy brosse un portrait original de Frank Scott par l'intermédiaire du regard de Jacques Ferron, qui lui a consacré des personnages phares de son œuvre littéraire. Elle présente, par ailleurs, les deux hommes comme des frères ennemis (Susan Margaret Murphy, Le Canada anglais de Jacques Ferron (1960-1970): formes, fonctions et représentations, préface de Jean Marcel, Québec, Les Presses de l'Université Laval, 2011).

12 Sur la confrontation entre Frank Scott et André Laurendeau, voir Guy Laforest "L'Accord du lac Meech : la recherche d'un compromis entre André Laurendeau et F. R. Scott ", dans Trudeau et la fin d'un rêve canadien, Sillery, Éditions du Septentrion, 1992, p. 81-119.

13 Cet article poursuit des réflexions amorcées dans notre thèse de doctorat (histoire), Penser et "panser" les plaies du Canada : le moment Laurendeau-Dunton, 19631971, Québec, Université Laval, 2013. 
multiplication des réflexions sur la dualité et aux tentatives de créer des rapprochements entre les "deux solitudes ». À l'origine de ces tentatives se trouve un réseau d'intellectuels qui œuvrera au sein de la commission Laurendeau-Dunton. Enfin, ce réseau sera abordé dans la dernière partie de l'article.

\section{Entre les repères identitaires britanniques et la crainte du voisin américain : construire une voie spécifiquement canadienne}

Les repères identitaires et symboliques du Canada se construisent, notamment, en constante relation avec deux pôles d'influence : l'Empire britannique et le voisin américain, voisin qui gagne en puissance au cours du $\mathrm{Xx}^{\mathrm{e}}$ siècle, devenant ainsi, aux yeux de certains Canadiens, une menace et pour d'autres, une source d'admiration. Autant ils s'abreuvent à ces pôles d'influence, autant des intellectuels vont élever leur voix pour promouvoir la création de nouveaux repères spécifiquement canadiens. Certains doutent également de la présence même d'un caractère spécifique au Canada et du potentiel de survie du Dominion. À ce titre, l'ouvrage de Goldwin Smith Canada and the Canadian Question est plutôt éloquent. Publié en 1891, il a l'effet d'une bombe au Canada anglophone ${ }^{14}$. Smith soutient que le Dominion constitue une absurdité géographique, ethnique et économique et que sa seule destinée repose sur une éventuelle union politique avec les États-Unis ${ }^{15}$. Ces propos vont choquer le Canada anglophone et susciter une réflexion sur la nature du pays et sur son caractère distinct. Cette crainte d'assister à la fin de l'aventure canadienne reviendra hanter ponctuellement les

${ }^{14}$ Damien-Claude Bélanger compare, d'ailleurs, l'effet choc causé par ce livre au Canada anglophone à celui qu'a eu le rapport Durham chez les francophones (Damien-Claude Bélanger, Prejudice and Pride: Canadian Intellectuals Confront the United States, 1891-1945, Toronto, University of Toronto Press, 2010, p. 9).

15 Ibid. 
intellectuels au cours de l'histoire du pays, alimentant alors les débats sur le nationalisme canadien et l'essence même du Canada.

Selon le sociologue Raymond Breton, ce n'est pas le fruit du hasard si la constitution du Canada a été nommée le British North America $A c{ }^{16}$. La nation qui se construit alors dans le Nouveau Monde ne le fait pas en rupture complète avec la mère patrie, comme ce fut le cas aux États-Unis. Au contraire, le Canada du XIX ${ }^{\mathrm{e}}$ siècle et du début du $\mathrm{xx}^{\mathrm{e}}$ siècle se veut davantage un prolongement des îles britanniques. Comme le mentionne Carl Berger, l'impérialisme est une forme de nationalisme canadien ${ }^{17}$. Il atteint son apogée de la fin du XIX ${ }^{\mathrm{e}}$ siècle à la période précédant la Première Guerre mondiale ${ }^{18}$. Ce nationalisme, qui s'appuie sur une critique virulente des ÉtatsUnis, est porté par un programme politique pensé par des intellectuels ${ }^{19}$ qui gravitent autour de certaines associations telles que l'Imperial Federation League et les Round Table Groups et diffusent leurs idées à travers le Queen's Quarterly, fondé en 1893, et le University Magazine, fondé en $1907^{20}$. Ces intellectuels souhaitent non seulement exalter les repères britanniques, mais aussi construire, à partir de ces repères, un Canada qui parviendrait à se différencier à l'intérieur de l'Empire et à négocier d'égal à égal avec lui. Les repères identitaires auxquels ils s'identifient sont alors étroitement liés à l'Empire et à ses symboles : l'Union Jack, la fierté rattachée au parlementarisme et la sympathie envers le personnage du gouverneur général. L'attachement aux symboles britanniques représente aussi une façon de se démarquer du voisin du Sud, où priment les principes de "life, liberty, and the pursuit of happiness". Au Canada, "la paix, l'ordre et le bon

16 Raymond Breton, "From Ethnic to Civic Nationalism: English Canada and Quebec ", Ethnic and Racial Studies, vol. 11, n 1 (janvier 1988), p. 88.

17 Carl Berger, The Sense of Power: Studies in the Ideas of Canadian Imperialism, 1867-1914, 2 éd., Toronto, University of Toronto Press, [1970] 2013, p. 259.

18 Voir Bélanger, Prejudice and Pride, p. 27.

19 Pour un portrait détaillé de ces intellectuels, voir le chapitre intitulé " Men » dans Berger, The Sense of Power, p. 12-48.

20 Bélanger, Prejudice and Pride, p. 25-26. 
gouvernement » font état d'un destin plus tranquille, moins empreint d'une tradition révolutionnaire. Comme l'explique Carl Berger, les impérialistes canadiens croient que le caractère des gens de leur pays, forgé par la droiture et la loyauté, forme un bouclier contre la décadence remarquée de l'autre côté de la frontière : "Running through their critique of republican society and their interpretation of the Canadian past was the belief that Canadians were pre-eminently a loyal and law-abiding people not given over the erratic and hectic behaviour of their neighbours ${ }^{21}$. "Or tous ne voient pas un rapprochement avec les États-Unis comme un pacte avec le diable. Sur la scène fédérale, les libéraux aspirent même à renforcer les liens économiques avec eux. Ils font d'ailleurs campagne aux élections de $1891^{22}$ et de 1911 en promettant de lever les barrières économiques entre le Canada et les États-Unis. Pour les conservateurs, attachés à la tradition, le libre marché avec le voisin américain ne fait qu'aviver le spectre de l'annexion. Il faut également souligner que l'attachement aux symboles britanniques ne suscite pas la même ferveur dans l'ensemble du pays. Au Canada français et au Québec, plus particulièrement, la sympathie envers l'Empire est plutôt faible ${ }^{23}$.

La Première Guerre mondiale et ses lendemains éprouvants assènent un coup violent à l'impérialisme. Les 60000 victimes canadiennes de la guerre, les négociations houleuses entre le Canada et la mère patrie pour l'obtention d'une plus grande autonomie ${ }^{24}$ et

21 Berger, The Sense of Power, p. 128.

22 Comme le mentionne Damien-Claude Bélanger au sujet de l'élection fédérale de 1891, "[t]he election pitted an ailing Macdonald and his National Policy and a youthful Wilfrid Laurier and his promises of unrestricted reciprocity with the United States" (Bélanger, Prejudice and Pride, p. 8).

23 Talbot, "Une réconciliation insaisissable ", p. 69. Talbot rappelle que certains impérialistes tenaient un discours faisant la promotion de l'assimilation des francophones. Ce genre de discours a certainement alimenté le rejet de l'impérialisme de la part des Canadiens français et leur peu d'attachement aux symboles britanniques.

${ }^{24}$ La coopération militaire entre les Britanniques et les Canadiens avait fait naître l'espoir d'une union plus équitable entre le Dominion et sa mère patrie (voir Berger, The Sense of Power, p. 264). 
les difficultés financières de l'Empire contribuent à calmer l'enthousiasme des impérialistes. Ruiné par les affres de la guerre, le Vieux Continent a perdu de son lustre. Le Canada se rapproche alors des États-Unis ${ }^{25}$. La culture populaire américaine et le vent de modernité qu'elle souffle exercent un fort pouvoir d'attraction et remplacent la culture populaire britannique ${ }^{26}$. Le Canada tisse également des liens économiques plus étroits avec son riche voisin. Or l'influence de la culture américaine et sa puissance économique ne sont pas sans soulever des inquiétudes chez certaines élites. Comme le souligne Doug Owram : "The very Canadian identity which had emerged from the shadow of Britain now seemed about to be overwhelmed by the rising power and influence of the United States ${ }^{27}$."

Avec le temps, les liens avec l'Empire s'étiolent, mais la volonté de se démarquer des États-Unis afin d'éviter l'annexion perdure. Le Statut de Westminster de 1931, qui signe l'indépendance du pays dans ses affaires étrangères, ne vient pas mettre un terme à l'attachement aux symboles britanniques au Canada. Comme le rappelle José Igartua : "English Canadian opinion continued in the 1950s to exhibit a strong attachment to Great Britain $[. . .]^{28}$. " Les débats déchirants entourant la création d'un drapeau spécifiquement canadien, des années 1940 aux années 1960, sont, à ce sujet, évocateurs ${ }^{29}$. Aux yeux de certains, surtout ceux d'allégeance conservatrice, le détachement des repères britanniques apparaît dangereux dans un contexte

\footnotetext{
Bélanger, Prejudice and Pride, p. 10.

${ }^{6}$ Ibid.

${ }^{7}$ Doug Owram, «Introduction », dans Berger, The Sense of Power, p. xIv.

${ }^{28}$ José Igartua, The Other Quiet Revolution: National Identities in English Canada, 1945-1971, Vancouver, University of British Columbia Press, 2006, p. 89.

29 Jusque dans les années 1950, le drapeau favori des Canadiens demeure l'Union Jack, comme le révèlent les résultats d'un sondage. D'ailleurs, depuis 1867 , le drapeau officiel du pays était celui de sa mère patrie, l'Union Jack. Il y avait certes le Red Ensign, drapeau non officiel, mais celui-ci était également lié à la Grande-Bretagne puisqu'il correspondait au drapeau de la marine marchande britannique, agrémenté du blason canadien dans un coin (voir Eva Mackey, The House of Difference: Cultural Politics and National Identity in Canada, Londres, Routledge, 1999, p. 55).
} 
où les États-Unis gagnent sans cesse en puissance et projettent une image parfois peu reluisante. En effet, les assassinats de personnalités politiques, les soulèvements causés par le mouvement des droits civiques, l'engagement dans le nucléaire ne font que renforcer la peur de voir le Canada s'intégrer dans ce pays secoué par de fortes turbulences ${ }^{30}$. Afin d'éviter l'annexion, certains militent pour que le Canada s'accroche à ce qui le distingue, sa tradition britannique. C'est notamment le cas de George Grant dans son essai Lament for a Nation, publié en 1965. Or le Canada auquel rêve et se réferre Grant appartient de plus en plus au passé. Ce Canada, attaché à la tradition britannique, n'a d'ailleurs jamais pu jeter un pont durable entre les anglophones et les francophones.

Au cours des années 1950 et 1960, deux conceptions du pays semblent s'affronter : l'une ancrée dans la tradition britannique, l'autre désireuse de projeter l'image d'un Canada nouveau, émancipé de ses anciens repères, capable de jouer un rôle clé sur la scène internationale et capable de se créer lui-même de nouveaux repères. Pour les générer, il fallait constituer un tout cohérent, s'entendre sur ce qui unissait les Canadiens et sur ce qui pouvait faire en sorte que l'aventure de la Confédération ne soit pas destinée à une fin abrupte se résumant à sa dissolution dans le tout américain. L'identification aux repères britanniques représentait de moins en moins une possibilité, surtout avec l'entrée en scène de Lester B. Pearson, qui devient premier ministre en 1963, à la suite d'une carrière de diplomate couronnée de succès et du prix Nobel de la paix en 1957. Libéral, Lester B. Pearson ne partage pas le fort attachement à l'héritage britannique de son opposant conservateur en Chambre, John Diefenbaker ${ }^{31}$. Il conçoit également que la clé de voûte du Canada est la volonté de rester uni. Pour atteindre cette union, il faut susciter un dialogue entre les deux peuples fondateurs, alors que de plus en

30 Owram, «Introduction ", p. XIV.

31 Sur l'attachement de Diefenbaker à la tradition britannique, voir Igartua, The Other Quiet Revolution, p. 129-130. 
plus d'éléments semblent les séparer. La cohésion nationale apparait comme un objectif à atteindre, surtout dans un contexte où le Canada cherche à occuper un rôle plus marqué sur la scène internationale ${ }^{32}$. $\mathrm{Si}$ le Canada parvient à transformer sa diversité porteuse de conflits en diversité créatrice, il pourra alors servir d'exemple sur la scène internationale. Aux anciens repères représentés par les symboles britanniques, d'autres font tranquillement leur apparition : le bilinguisme, le biculturalisme, l'exaltation du dialogue et la volonté d'aller à la rencontre de l'Autre. Certes, tous n'adhèrent pas à ce Canada, mais de plus en plus d'intellectuels, d'universitaires et de responsables politiques en vantent les mérites.

\section{Affirmation d'une volonté de créer des ponts : ouverture d'un dialogue entre les deux peuples fondateurs}

Depuis le début de la Confédération canadienne, la cohabitation entre les différentes communautés culturelles du pays et, plus spécifiquement, entre le Canada français et le Canada anglais fait l'objet de défis importants. Les relations entre les francophones et les anglophones sont ponctuées de tensions cristallisées lors d'événements comme les deux crises de la conscription. Avec l'avènement d'un nationalisme plus revendicateur dans le Québec des années 1960, le mur qui séparait les " deux solitudes " semble de plus en plus infranchissable. Des intellectuels appellent alors au rétablissement de canaux de communication entre les deux peuples

\footnotetext{
Sur le rôle international du Canada dans les années 1960, voir Kim Richard Nossal, Stéphane Roussel et Stéphane Paquin, Politique internationale et défense au Canada et au Québec, Montréal, Les Presses de l'Université de Montréal, 2007, p. 109116. Les auteurs utilisent le terme de puissance moyenne pour décrire le nouveau rôle qu'aspire à occuper le Canada sur la scène internationale au lendemain de la Seconde Guerre mondiale : "Concrètement, cette politique se traduit par une diplomatie très active, l'adoption du rôle de médiateur et de conciliateur, et une participation presque systématique aux opérations multilatérales destinées à assurer la paix. Bien entendu, les puissances moyennes ne sont pas les seules à adopter de tels comportements. Mais elles tendent à le faire de façon plus systématique que les autres catégories d'États" (Ibid., p. 116).
} 
fondateurs afin d'endiguer d'éventuelles tensions à même de menacer la survie du pays. Si la prise de conscience d'une crise intérieure se fait surtout dans les années 1960, elle se présente bien avant et s'exprime en littérature, notamment avec la publication du roman emblématique d'Hugh MacLennan Two Solitudes, en 1945. Elle s'exprime aussi à travers la recherche universitaire, à travers la création de livres ou de revues comme Exchange, qui se veulent un espace de dialogues, et à travers des discours plus personnels. Ces initiatives, tantôt modestes, tantôt ambitieuses, tantôt couronnées de succès, tantôt infructueuses, sont intéressantes, car elles témoignent d'une époque où la dualité devient un objet d'étude et où la volonté de voir émerger un dialogue se présente autant chez les intellectuels et les universitaires que chez les responsables politiques.

\section{De l'ambition à la modestie : le cas du Comité pour l'étude des deux cultures/Committee for Biculturalism}

Parmi ces initiatives, se retrouvent notamment des ouvrages d'universitaires portant sur la dualité, le bilinguisme et les relations canado-québécoises. Ces ouvrages, qui semblent se multiplier dans les années de l'après-guerre, témoignent de cet intérêt croissant pour comprendre le Canada, interroger son passé afin de mieux préparer son avenir. Ils révèlent également cette volonté de repenser les relations entre les deux communautés culturelles principales du pays afin que la dualité, thématique récurrente en littérature dans les années précédant la commission Laurendeau-Dunton, soit mieux respectée.

En 1945, le Conseil de recherche en sciences sociales du Canada demande à un comité d'experts si une étude poussée sur l'état des relations canado-québécoises est envisageable. La réponse affirmative donne naissance à un comité auquel siègent trois professeurs : le sociologue Jean-Charles Falardeau, l'historien Arthur Lower et l'économiste Burton Seely Keirstead. Composé d'universitaires renommés, ce comité trace les premiers jalons d'initiatives similaires qui suivront 
dans le milieu universitaire, en rassemblant à la fois des anglophones et des francophones et en mettant à profit plusieurs disciplines des sciences sociales, alors en plein déploiement ${ }^{33}$. Au départ, l'expérience se veut vaste et couvre plusieurs enjeux. Elle doit mener à une étude exhaustive de

l'histoire récente du " mariage de raison " canadien; [des] conditionnements écologiques et démographiques des relations sociales; [des] institutions et [des] partis politiques en tant que cadres d'affrontement, de discussion et de compromis; [des] processus de la vie économique; [des] échanges socio-culturels et [des] conséquences psychologiques résultant de la distance sociale, des contacts et des tentatives de communication ${ }^{34}$.

Bref, les chercheurs aspirent à brosser un portrait détaillé de ce qui pourrait expliquer la distance entre le Québec et le reste du Canada. Ils emploient la métaphore de " mariage de raison " pour aborder les relations canado-québécoises et cherchent, en quelque sorte, à savoir pourquoi cette alliance semble parfois si peu naturelle. Le projet, intitulé "Comité pour l'étude des deux cultures ou Committee for Biculturalism ", reflète jusque dans le bilinguisme de son titre ce souci d'équilibre, cette volonté de susciter une collaboration entre les deux peuples fondateurs. Devant l'ampleur de la tâche à accomplir, l'exaltation des débuts s'évanouit et le projet est ramené à des proportions plus modestes. Comme en témoigne Jean-Charles Falardeau : «Ce qui se passa durant les mois, durant les années qui

33 Sur le développement des sciences sociales au Québec, voir Jean-Philippe Warren, L'engagement sociologique : la tradition sociologique du Québec francophone (18861955), Montréal, Éditions du Boréal, 2003.

34 Jean-C. Falardeau, "Avant-propos ", dans Mason Wade (dir.), La dualité canadienne : essais sur les relations entre Canadiens français et Canadiens anglais = Canadian Dualism: Studies of French-English Relations, avec la collaboration d'un comité du Conseil de recherche en sciences sociales du Canada sous la direction de Jean-C. Falardeau, Québec, Les Presses de l'Université Laval; Toronto, University of Toronto Press, 1960, p. vi, [En ligne], [http://dx.doi.org/doi:10.1522/030276428]. 
suivirent, ressembla davantage à une comédie italienne qu'à une symphonie minutieusement prévue ${ }^{35}$."

Une des raisons qui explique le caractère erratique du projet et la révision à la baisse des objectifs initiaux est sans doute l'état lamentable de la recherche universitaire sur des sujets similaires. Cet état était tel que la multiplication de collaborateurs de haut calibre, tels que l'économiste Harold Innis ou le directeur des Archives nationales William Kaye Lamb, n'a pas suffi à donner l'impulsion voulue au comité ${ }^{36}$. Jean-Charles Falardeau explique les hauts, mais surtout les bas de ce projet prometteur : "Six années s'écoulèrent ainsi, ponctuées d'arrivées et de départs, de démarrages et de retraites. À l'hiver de 1954, nous dûmes reconnaître que notre plan original était téméraire dans l'état actuel de la vie académique canadienne. Il fallait renoncer à notre vaste plan d'enquête et restreindre notre effort à une tentative plus modeste et immédiatement réalisable ${ }^{37}$. " L'enquête exhaustive, qui devait couvrir plusieurs terrains et se pencher autant sur les raisons économiques, démographiques que culturelles de la distance entre les deux peuples fondateurs, donne finalement naissance à un ouvrage collectif dirigé par le chercheur américain Mason Wade, réunissant une vingtaine de contributeurs. « Dira-t-on que la montagne a enfanté d'une souris? ", s'interroge Jean-Charles Falardeau. Peutêtre. Toutefois, malgré l'incapacité des chercheurs à atteindre les objectifs fixés au départ, il n'en reste pas moins que ce collectif, de par le nombre élevé de collaborateurs qui y ont contribué, crée une émulation dans le milieu universitaire et contribue à faire de l'étude des deux communautés culturelles principales du Canada un sujet d'intérêt dans plusieurs universités. Jean-Charles Falardeau souligne, dans la préface de La dualité canadienne/Canadian Dualism, les plus importants projets se rattachant au champ des relations canadoquébécoises, parmi lesquels se trouvent :

$\begin{array}{ll}{ }_{35} & \text { Ibid. } \\ { }_{3}^{36} & \text { Ibid. } \\ & \\ 37 & \text { Ibid. }\end{array}$ 
les recherches sur les composantes psycho-sociales des relations inter-ethniques poursuivies sous la direction du P. Noël Mailloux o.p. par l'Institut de Recherches en Relations humaines de Montréal; l'enquête sur les perspectives culturelles de l'enseignement élémentaire canadien-français entreprise, depuis quelques années, par l'École de Pédagogie de l'Université Laval. À ces travaux de longue haleine il faut ajouter de nombreuses études subventionnées par le Comité psychologique et sociologique du Conseil de Recherche pour la Défense, en particulier celles des professeurs J. M. Blackburn et Andrew Kapos de l'Université Queen's, et les travaux exécutés pour le compte des Laboratoires médicaux du Conseil de Recherche pour la Défense par le professeur David N. Solomon et J. Jacques Brazeau ${ }^{38}$.

\section{Le Congrès des affaires canadiennes}

L'ouvrage collectif dirigé par Mason Wade ne constitue pas un exemple isolé. En 1961, le premier Congrès des affaires canadiennes est mis sur pied; il s'intéresse à la survie du Canada dans le contexte de la montée en puissance des États-Unis. Il constitue le premier événement d'une série de rencontres qui donnent lieu à la création de la collection "Congrès des affaires canadiennes " aux Presses de l'Université Laval. Les titres des ouvrages publiés sont évocateurs du malaise ressenti à une époque où l'heure est au bilan, après presque un siècle de cohabitation : Le Canada, expérience ratée... ou réussie? (1962), L'économie canadienne : où allons-nous? (1962), Les nouveaux Québécois (1963) et La dualité canadienne à l'heure des États-Unis (1965). Le Congrès possède un caractère résolument bilingue et rassemble autant des anglophones que des francophones. Au même titre, ses publications sont bilingues.

Parmi les collaborateurs de la première édition du Congrès tenue du 15 au 18 novembre 1961 à l'Université Laval, se trouvent autant des responsables politiques que des universitaires et des journalistes. Jean Lesage, E. Davie Fulton, René Lévesque, Murray G. Ballantyne,

38 Ibid., p. VII-VIII. 
Jean-Jacques Bertrand, Marcel Chaput, Douglas Fisher, Eugene Forsey, Maurice Lamontagne, André Laurendeau, James R. Mallory, Michael Oliver, Gérard Pelletier et Mason Wade forment la liste des invités ${ }^{39}$. Il semble se dégager des conférences présentées lors du Congrès une volonté de faire le bilan de l'aventure canadienne, de soulever les écueils rencontrés et de trouver des solutions pour l'avenir. Lévénement organisé par l'Association générale des étudiants de l'Université Laval montre à quel point les réflexions sur l'avenir du Canada touchent toutes les générations; mise sur pied par des étudiants, la rencontre rejoint des collaborateurs nés dans les années 1900, 1910, 1920 et 1930. La présence d'invités prestigieux, tels que le premier ministre québécois Jean Lesage ou le ministre de la Justice canadien E. Davie Fulton, est également révélatrice de l'ouverture à la réflexion présente à l'époque. Le but poursuivi par le Congrès est notamment de désamorcer les préjugés. Comme le relate l'avantpropos, la rencontre permet de changer les perceptions de ses participants :

Les délégués de langue anglaise ont été plongés dans un milieu qui témoigne activement de la révolution intellectuelle qui agite le Canada français. Pour certains, c'était leur première rencontre avec le Canada français. Ils ont dû prendre conscience, parfois brutalement, des difficultés auxquelles les Canadiens anglais ou français, ont à faire face, dans le système confédératif, et à la tendance séparatiste qui apparaît, à certains Canadiens français, comme une solution plus réaliste que la Confédération.

Beaucoup de délégués de langue française ont pu, de leur côté, se rendre compte que la réputation du Canada anglais, d'entretenir à l'endroit du Québec un sentiment d'apathie ou d'antipathie, n'est

39 Parmi les organisateurs de l'événement se trouvent Peter White et Brian Mulroney. Loin de constituer un coup d'épée dans l'eau, cette conférence est l'objet de plusieurs publications dans les journaux (voir Jean-Jacques Bertrand, " La Confédération : en théorie... et en pratique ", dans Association des étudiants de l'Université Laval, Le Canada, expérience ratée... ou réussie? Le Congrès des affaires canadiennes, 1961 = Canadian Experiment, Success or Failure? Congress on Canadian Affairs, 1961, Québec, Les Presses de l'Université Laval, 1962, p. 148-149). 
pas tout à fait justifiée, et que son ignorance du Canada français n'est pas aussi flagrante qu'ils avaient tendance à le croire ${ }^{40}$.

L'avant-propos se conclut par l'éloge du dialogue, panacée au mal canadien : " [...] nous savons mieux, maintenant qu'un dialogue franc et ouvert permet d'attendre beaucoup de l'avenir. Nous voudrions que les prochains Congrès permettent la poursuite d'un tel dialogue ${ }^{41}$.»

Dans son allocution inaugurale, le ministre de la Justice et procureur général du Canada, E. Davie Fulton, revient sur un thème omniprésent dans les discours de l'époque : la nécessité de consolider les assises de la nation canadienne afin d'éviter l'américanisation. Une des façons de contrer cette assimilation réside dans la reconnaissance du partenariat égal entre les deux peuples fondateurs. Omniprésentes dans l'ouvrage collectif, les thématiques de la dualité et du bilinguisme teintent son discours. Il insiste également sur la nécessité pour le Canada de grandir pour devenir une "nation adulte ${ }^{42}$ ", comme si le pays, alors en crise d'adolescence, devait se soumettre à une réflexion poussée pour passer à l'âge adulte et faire face aux défis de l'avenir. Il explique ainsi les dangers qui menacent le Canada :

Aujourd'hui encore, est-ce que l'influence économique et culturelle des États-Unis a diminué? La montée en flèche des placements de capitaux américains depuis 1920 et particulièrement depuis la seconde guerre, nous fournit une réponse éclatante du contraire. Ces investissements ont été effectués partout au Canada, y compris au Québec.

Que dire maintenant de l'influence de la culture, de la pensée, du mode de vie américain? L'Europe entière la ressent, pourtant l'océan l'en sépare. Que dire de nous qui captons régulièrement les émissions de radio et de télévision? Telles sont les conditions difficiles dans lesquelles nous nous trouvons pour bâtir une nation canadienne. L’enjeu est énorme, et, d'une certaine façon, les Anglo-

\footnotetext{
40 "Avant-propos », dans Ibid., p. 6.

41 Ibid.

${ }^{42}$ E. Davie Fulton, "La Confédération, un succès... et un défi », dans Ibid., p. 18.
} 
canadiens courent un risque plus grand à cause de leur affinité de langage avec les États-Unis ${ }^{43}$.

Afin de préserver les bases de la Confédération canadienne, E. Davie Fulton souligne l'importance de s'adonner à un examen de conscience, autant pour les Canadiens anglais que pour les Canadiens français, afin de prendre connaissance de leurs faiblesses et d'y remédier. Il appelle à une plus grande ouverture à l'Autre de la part de "la génération Anglo-Canadienne ". Selon lui, elle " doit comprendre les Canadiens français qui exigent un statut égal dans la Confédération et connaître leur culture et leurs traditions qui sont essentiellement différentes des nôtres. Dans plusieurs cas, les griefs des Canadiens français sont tout à fait justifiés ${ }^{44}$ ". Selon son analyse, la dualité s'inscrit dans l'ADN du pays; il remarque d'ailleurs une certaine évolution dans l'attitude des Canadiens anglophones, plus ouverts à accepter cette dualité qu'il y a quelques années : "Les Anglo-Canadiens d'aujourd'hui acceptent davantage notre dualité ethnique et ses conséquences. [...] Ils découvrent l'importance du Canada français et le rôle vital qu'il joue dans le façonnement de notre pays ${ }^{45}$. " Afin de poursuivre l'affirmation de cette dualité, la connaissance de l'Autre et le bilinguisme deviennent des conditions essentielles, surtout pour les Anglo-Canadiens qui sont en contacts fréquents avec leurs homologues francophones : «L'essentiel dans les années à venir, c'est d'obtenir des Anglo-Canadiens qui sont appelés à collaborer régulièrement avec les Canadiens français, qu'ils comprennent la langue, la culture et les institutions de ces derniers. [...] Si je semble tant insister sur l'importance du bilinguisme, tel que je l'entrevois, c'est que j'ai la conviction profonde que c'est la voie à suivre pour donner à notre nation une dimension adulte ${ }^{46}$. "

43 Ibid., p. 12-13.

44 Ibid., p. 14.

45 Ibid., p. 16.

46 Ibid., p. 18. 
Cet appel au dialogue, à l'ouverture à l'Autre, à la curiosité lancé par E. Davie Fulton, un anglophone de la Colombie-Britannique, témoigne de ce changement des mentalités qui s'opère au tournant des années 1960. Le reste de l'ouvrage témoigne d'une synchronie intéressante : tous les sujets abordés le sont par un francophone et un anglophone afin de mettre en lumière les deux optiques. À la conférence de Murray G. Ballantyne intitulée "What French Canadians have Against us? ", Gérard Pelletier répond avec la présentation suivante : «Les Canadiens anglais nous reprochent... ». Toutes les conférences présentées en français sont traduites en anglais et vice-versa. Bref, ces actes du Congrès des affaires canadiennes traitent du dialogue et constituent en soi un dialogue qui se veut le plus équitable possible. Les organisateurs et les éditeurs semblent avoir tenté de reproduire à petite échelle leur Canada idéal : un Canada faisant une juste part à chacun des deux peuples fondateurs, et ce, en dépit des divergences d'opinions.

Le Congrès se clôt - avant l'allocution de fermeture du premier ministre du Québec Jean Lesage - par une table ronde portant le même titre que l'ouvrage : «Le Canada : expérience ratée... ou réussie? » Mason Wade dirige cette séance où sont conviés Jean-Jacques Bertrand, Douglas Fisher et Maurice Lamontagne. Unilingue anglophone, Douglas Fisher, député fédéral du CCF (Co-operative Commonwealth Federation) du comté de Port-Arthur en Ontario, offre une perspective divergente de celle de ses collègues en soulignant le vif succès de l'expérience de la Confédération. Pour lui, les velléités séparatistes du Québec représentent une aberration, et le provincialisme ne constitue pas la solution aux défis économiques de l'avenir ${ }^{47}$. L’antiaméricanisme représente également une attitude juvénile. Pourquoi ne pas tirer le meilleur de la culture américaine?, se demande-t-il ${ }^{48}$.

47 Douglas Fisher, "The Average English Canadian View ", dans Ibid., p. 157-159.

48 Ibid., p. 158. 
D’un optimisme mesuré, l'économiste Maurice Lamontagne ${ }^{49}$ soutient que la Confédération canadienne constitue certes une réussite, mais qu'elle ne peut plus continuer dans sa forme actuelle. Elle doit être repensée pour témoigner de «l'évolution récente et des réalités nouvelles ${ }^{50}$ ". Cette évolution récente est marquée par la recherche d'autonomie des Canadiens français et par l'ouverture des Canadiens anglais à l'Autre. Il souligne que la cause du malaise séculaire observé par des citoyens et des intellectuels au Canada est d'ordre culturel. Il rappelle les origines historiques de ce malaise : «Dès le lendemain de la Confédération, les relations entre les deux groupes ont été caractérisées par un climat de guerre froide et même de vives querelles. [...] Après la première guerre mondiale, ce fut la période de coexistence sans rencontres, les deux groupes vivant leur propre vie en s'ignorant plus ou moins ${ }^{51}$. " Avec l'apparition d'un Canada français plus confiant, qui ne remet plus en question sa survie, l'équilibre des forces doit changer à son avis. La reconnaissance symbolique du Canada français au sein de la Confédération canadienne ne suffit plus. La solution réside, selon l'analyse du politologue québécois, dans l'établissement d'un Canada qui respecte et reconnaît l'apport des deux cultures, " un Canada bi-culturel ${ }^{52}$ ». Il décrit alors ce qu'il entend par Canada biculturel : «Il ne s'agit pas évidemment de demander à chaque Canadien de devenir parfaitement bilingue et de participer également à nos deux cultures. Il faut toutefois établir

49 Maurice Lamontagne a dirigé le Département d'économie de la Faculté des sciences sociales de l'Université Laval avant de quitter le département en 1954 et de s'installer à l'Université d'Ottawa. En 1954, il publie le Fédéralisme canadien, ouvrage consacré à un nouveau fédéralisme qui prône une centralisation modérée et un meilleur dialogue entre les francophones et les anglophones (Maurice Lamontagne Le fédéralisme canadien : évolution et problèmes, Québec, Les Presses de l'Université Laval, 1954).

50 Maurice Lamontagne, "Rechercher l'union véritable des deux cultures ", dans Association des étudiants de l'Université Laval, Le Canada : expérience ratée... ou réussie?, p. 163.

51 Ibid., p. 165.

52 Ibid. 
des foyers de bi-culturalisme et faciliter à tous les Canadiens l'accès à nos cultures ${ }^{53}$."

Il propose alors un programme concret, qui n'est pas sans rappeler des éléments phares du programme de la commission LaurendeauDunton. Maurice Lamontagne suggère de renforcer la présence des Canadiens français dans les postes de gestion des entreprises établies au Québec. À son avis, il faudrait également que les anglophones occupant des postes de gestion dans ces entreprises puissent s'exprimer en français. L'enseignement de la langue seconde, autant pour les francophones que pour les anglophones, doit être rehaussé. Le bilinguisme devrait occuper une plus grande place non seulement dans les écoles et dans les hautes sphères de la gestion, mais également dans la fonction publique fédérale. Enfin, le programme imaginé par Lamontagne touche également au monde de la communication : "Les réseaux anglais et français de radio et de télévision devraient être complétés de façon à permettre à la très grande majorité des Canadiens de prendre contact avec nos deux principales cultures ${ }^{54}$."

Ce n'est pas le fruit du hasard si la lecture de ce programme visant à insuffler un caractère biculturel au Canada fait penser au mandat de la commission Laurendeau-Dunton ${ }^{55}$. Maurice Lamontagne, un des principaux conseillers de Lester B. Pearson, a lui-même écrit ce mandat, comme en témoigne Léon Dion : " [Maurice Lamontagne] convainc le premier ministre Pearson de créer la Commission royale d'enquête sur le bilinguisme et le

${ }^{53}$ Ibid.

${ }^{5}$ Ibid., p. 164.

55 Le mandat de la Commission était de « [f]aire enquête et rapport sur l'état présent du bilinguisme et du biculturalisme au Canada et de recommander les mesures à prendre pour que la Confédération canadienne se développe d'après le principe de l'égalité entre les deux peuples qui l'ont fondée, compte tenu de l'apport des autres groupes ethniques à l'enrichissement culturel du Canada, ainsi que des mesures à prendre pour sauvegarder cet apport " (Commission royale d'enquête sur le bilinguisme et le biculturalisme, Rapport préliminaire de la Commission royale d'enquête sur le bilinguisme et le biculturalisme, Ottawa, Imprimeur de la reine, 1965, p. 143). 
biculturalisme, rédige l'essentiel de son mandat et persuade André Laurendeau d'en accepter la coprésidence avec Davidson Dunton ${ }^{56}$. "

La notion de biculturalisme occupe de plus en plus de place dans l'espace public au cours des années 1960. Ce biculturalisme, il ne peut être atteint que par le dialogue. Dans l'ouvrage intitulé La dualité canadienne à l'heure des États-Unis, Raymond Morel, président de la quatrième édition du Congrès des affaires canadiennes, revient sur l'importance de contribuer à tisser des liens entre les deux communautés culturelles principales :

Depuis sa création, le Congrès des Affaires canadiennes a voulu favoriser le dialogue des deux nations canadiennes sur un plan d'égalité rigoureuse. C'est ce qui a encore été tenté au cours de ce quatrième congrès.

Le problème fondamental pour tout pays réside dans son existence même comme collectivité poursuivant des intérêts propres, vivant d'une économie et d'une culture qu'il a faites siennes par des efforts soutenus. De ce point de vue, le Canada n'a d'existence que dans l'esprit des géographes. Rien, jusqu'ici, n'a pu faire que les Canadiens se rejoignent dans des convictions communes, que des liens indéfectibles les unissent, bref, qu'ils aient claire conscience de leur raison d'être.

Car enfin, pourquoi être Canadiens quand tout en nous et autour de nous parle américain? Notre politique étrangère, notre économie, nos syndicats, nos modes de vie reflètent l'omniprésence américaine chez nous comme nulle part ailleurs.

C'est en somme, notre question : à quand le Canada aux Canadien $^{57}$ ?

${ }^{56}$ Léon Dion, Québec 1945-2000 : t. II : Les intellectuels et le temps de Duplessis, Sainte-Foy, Les Presses de l'Université Laval, 1993, p. 182.

57 Raymond Morel, "Avant-propos ", dans La dualité canadienne à l'heure des ÉtatsUnis : IVe Congrès des affaires canadiennes, Québec, Les Presses de l'Université Laval, 1965, p. 5. 
Cet avant-propos se veut particulièrement éloquent quant au contexte dans lequel prend forme la commission Laurendeau-Dunton, un moment où les élites intellectuelles, les universitaires plus particulièrement, mais aussi des responsables politiques, prennent conscience du problème canadien; un moment où ils aspirent à redonner le Canada aux Canadiens, à trouver des référents communs forts pour souder les liens entre les Canadiens. "Le Canada n'a d'existence que dans l'esprit des géographes ", voilà une phrase-choc qui résume le climat d'incertitude des années 1960. Ce climat est nourri par le besoin des intellectuels et des responsables politiques de réaffirmer le nationalisme canadien afin de consolider l'expérience canadienne à l'aube de son premier centenaire. Devant le nouveau rôle qu'occupe le Canada sur la scène internationale et devant les préoccupations concernant le transfert de pôles d'influence qui passent progressivement de l'Europe aux États-Unis, les élites canadiennes sentent le besoin de s'interroger sur le passé et le présent du pays pour construire un avenir meilleur. Certes, l'avant-propos dont il est question ici est publié une fois la commission Laurendeau-Dunton mise en branle, mais il témoigne des doutes que soulève la possible pérennité de l'expérience canadienne et du besoin d'agir avant qu'il ne soit trop tard.

Le collectif se conclut par la présentation de Paul Gérin-Lajoie, qui souligne que l'avenir du pays passe par l'expression de sa dualité : "Dans mon esprit, le Canada à l'heure des États-Unis doit donc avant tout, comme vous avez eu raison de le signaler par la thèse de votre congrès, se concevoir comme "dualité canadienne". Le Canada à l'heure des États-Unis doit être, de toute évidence, est, dans le sens que je viens de le décrire, un Canada à l'heure du Québec ${ }^{58}$. "

58 Paul Gérin-Lajoie, "La dualité canadienne à l'heure du Québec ", dans Ibid., p. 132. 


\section{Le cas de la revue Exchange et quelques articles dans d'autres revues}

À l'instar de La dualité canadienne, plusieurs projets aspirant à jeter des ponts entre les deux solitudes et à mieux les comprendre voient leur ambition initiale déçue. La motivation est palpable, autant chez les anglophones que chez les francophones, les efforts sont présents, mais les résultats escomptés ne sont pas toujours atteints. Un de ces efforts est représenté par la création de la revue Exchange, née dans le sillage de la commission O'Leary, qui met en lumière la nécessité de créer des magazines spécifiquement canadiens ${ }^{59}$. Dirigée par un éditeur européen, Stephen Vizinczey, la revue est basée à Montréal. Elle aspire à rassembler des auteurs canadiens des deux langues principales afin de créer une revue biculturelle et bilingue permettant de rapprocher les deux grands groupes culturels du Canada en discutant de politique, de littérature et de culture. Publiée pour la première fois le $1^{\text {er }}$ novembre 1961, la revue présente une ligne éditoriale claire en ce qui a trait à ses aspirations biculturelles. La formulation "There is a need... » ouvre chaque paragraphe, rythmant l'éditorial de cette répétition et rappelant ainsi le pressant besoin de

59 Aussi appelée Royal Commission on Publications, la commission O'Leary est mise sur pied en septembre 1960 afin d'étudier l'influence de la presse étrangère sur la presse nationale canadienne, dans un contexte de définition des contours identitaires de la nation canadienne. Pour un bref résumé des enjeux soulevés par la Commission, voir Richard Stursberg, "Royal Commission on Publications », 7 février 2006, [En ligne], [http://www.thecanadianencyclopedia.ca/en/article/royalcommission-on-publications] (15 juin 2014). Voir aussi l'éditorial d'Exchange, qui revient sur les circonstances de sa naissance : "If we succeed, much of the credit must go to those who already serve similar aims with the CANADIAN FORUM, with THE TAMARACK REVIEW and at the C.B.C. Moreover, if any new Canadian magazine will succeed, credit must go to Mr. O'Leary and his commission; their hearings and report did a great deal to awaken Canadians the need for national publications. We offer here our sincere thanks to all of them. I In launching EXCHANGE we may well over-estimate our ability to create the king of magazine the country needs - and we may well over-estimate the forthcoming sympathy and support. We are not certain of our success, but we are certain of the need" ("Éditorial ", Exchange: a Canadian Review, vol. 1, no 1 (novembre 1961), p. 1). 
donner vie à un tel projet afin de tisser des liens pérennes entre les Canadiens :

There is a need for a new Canadian magazine which expresses the bicultural reality of Canada and publishes the best English and French writers in the country. There is a need for a new Canadian magazine that provides both a link and a dividing line between Canadians and the rest of mankind - a magazine that helps the realization of individual and national identity.

There is a need in Canada for a new political magazine that provides a free exchange of views on our moral, social and economic problems. There is a need in Canada for a new political magazine that is guided by the belief that the challenge of the free flow of opinions is vital to the mental and social well-being of our nation - a magazine that is guided by moral principles instead of ideologies or the principle of expediency.

There is a need in Canada for a new literary magazine that can prove to the reader that good literature is not a bore. There is a need in Canada for a new literary magazine that published writers of talent and daring, whether they are known or unknown and regardless of what they are trying to say and in what style and form.

There is a need for a magazine of courage and integrity - a magazine which is ready to please no one and offend everyone but the innocent $[\ldots]^{60}$.

Le premier numéro de la revue consacre d'ailleurs un dossier au Québec, intitulé "The Grievances of French Quebec ». Les doléances des Québécois y sont explorées dans huit articles ${ }^{61}$. Ces derniers ne constituent pas des inédits; ils ont été traduits du français à l'anglais afin de leur donner une plus grande réception chez les anglophones.

60 Ibid., p. 1.

${ }^{61}$ Pour la table des matières détaillée du numéro, voir Ibid., p. 7. Outre les articles mentionnés, il y a un portrait de Pierre Jérôme (p. 50) et un article de Léon Dion, "The Old and the New Regime ", p. 45, déjà paru en français sous le titre «De l'ancien au nouveau régime " dans Cité libre, vol. 12, n³8 (juin-juillet 1961), p. 3-14, [En ligne], [http://collections.banq.qc.ca/ark:/52327/2225388]. 
Les lecteurs d'Exchange peuvent donc prendre connaissance des débats enflammés qui animent le Québec en lisant un extrait du manifeste du Rassemblement pour l'indépendance nationale (RIN) ou, encore, quelques passages des Insolences du Frère Untel. Le dossier portant sur la province francophone témoigne également de la diversité des genres littéraires présents dans les pages d'Exchange afin de faire découvrir la culture d'une solitude à l'autre : il y a une bande dessinée sur la corruption, une satire de Jacques Ferron intitulée « The Outraged Biped ", qui se moque du personnage de l'Anglo-Canadien ${ }^{62}$, et un échange musclé entre André Laurendeau et Pierre Bourgault au sujet de la montée du séparatisme au Québec. En lisant ce magazine, les anglophones entrent en contact avec ces figures de proue du Québec; ils peuvent découvrir cet Autre qu'ils connaissent si peu en raison de la distance ou de la barrière linguistique. Ils peuvent constater que le Québec ne constitue pas un tout homogène, qu'il y a des divergences d'opinions, notamment entre André Laurendeau et Pierre Bourgault, sur les façons d'entrevoir l'avenir, le premier privilégiant un fédéralisme renouvelé, le second l'indépendantisme ${ }^{63}$. La traduction représente ici un effort de rapprochement.

${ }^{62}$ Voici un extrait du texte de Jacques Ferron illustrant le ton sarcastique employé pour dénoncer l'égoïsme de certains Canadiens anglais, qui n'arrivent pas à regarder au-delà de leur nombril : "A self-respecting Englishman marvels at the fact that he walks on his hind legs, though he never boasts about it. Perhaps he is rather shy and troubled by the pure, primitive side of his nature. [...] The company of animals does not displease him, but while the African or the Latin admires in them their passionate vitality and the splendour of their mating games, the Englishman wants them only to bear witness and disdains the rest; he likes them to see him standing, that is all. He is touched and reassured, consoled for being an Englishman. The other biped have always troubled him. He could never understand how they could walk erect without speaking English. [...]” (Ibid., p. 63).

${ }^{63}$ Le débat entre les deux hommes est d'ailleurs présenté comme un dialogue entre deux générations, la génération de Bourgault, des jeunes nés dans les années 1930 et qui sont de plus en plus nombreux à croire que l'avenir du Québec doit se construire en dehors du Canada, et la génération d'André Laurendeau, née dans les années 1910, qui croit que le dialogue peut mener à une réconciliation durable entre le Québec et le Canada (Ibid., p. 57-62). 
La revue récolte de bons commentaires. The Montreal Star souligne, dans un article consacré à la parution du premier numéro de la revue : "Magazines like this are badly needed "64. S. S. Lamb, de la Canadian Broadcasting Corporation (CBC), souligne, pour sa part, la grande qualité du dossier consacré au Québec:

In the first category, there is a lengthy section on the grievances of French Canada. Articles of this sort are usually a token value, admission of the bad conscience on the part of the Anglo-Canadian editor. But the series in Exchange is of real interest, giving five French Canadians ample space to speak for themselves. In fact, it is some of the best introductions to the Quebec situation I have seen ${ }^{65}$.

Michael Oliver se fait également élogieux : «Bravo for Exchange. Bright yet serious; attractive without being slick; critical but never just carping - these are the impressions for the first two issues. I have always thought that a Montreal review could bring to Canadians the best in current thinking from both French and English communities. Exchange is the striking proof that this is true. Very best wishes for your success in this important and exciting venture ${ }^{66}$."

Malgré le succès relatif remporté par Exchange et les commentaires positifs envoyés par le lectorat, on doit mettre fin au projet après trois numéros seulement. La revue a certes réussi à générer 1700 abonnements, mais sa présentation recherchée, où textes et images artistiques ou bandes dessinées s'entremêlent, coûte une fortune à produire. Les fonds viennent donc rapidement à manquer ${ }^{67}$.

Le cas d'Exchange est particulièrement intéressant puisque la revue a été créée spécifiquement pour jeter un pont entre les anglophones et les francophones. Toutefois, au début des années 1960, des articles sont publiés dans plusieurs revues canadiennes, notamment dans The Canadian Forum et dans le magazine Maclean's, pour

\footnotetext{
${ }^{64}$ Exchange, vol. 1, n 2 (décembre 1961), p. 64.

65 Ibid.

${ }^{66}$ Exchange, vol. 2, nº 3 (février-mars 1962), p. 1.

${ }^{67}$ Ibid., p. 80.
} 
demander à ce que les mentalités changent et à ce que les Canadiens fassent preuve d'ouverture envers l'Autre. Il est impossible ici de faire le bilan exhaustif de tous ces articles, mais citons simplement quelques exemples. En novembre 1962, Eugene Forsey, qui a participé au Congrès des affaires canadiennes, où il a rencontré André Laurendeau, publie un article dans la Revue canadienne d'économique et de science politique. Cet article se veut la transcription de l'allocution qu'il a présentée lors de la rencontre annuelle de l'Association canadienne de science politique. Signe que le bilinguisme est dans l'air du temps, Forsey prononce son discours dans les deux langues officielles du Canada et annonce le nouveau caractère bilingue de l'Association. Intitulée "Canada: Two Nations or One? ", sa présentation rejette l'idée de reconnaître deux nations au pays, mais souligne tout de même l'importance de s'ouvrir à un nouveau partenariat plus équitable. À ses yeux, les anglophones doivent commencer à apprendre le français et à s'intéresser à la culture francophone. D'un autre côté, les francophones doivent également s'ouvrir au Canada anglophone et arrêter de le voir comme un tout monolithique. Dans les deux cas, les préjugés doivent cesser de triompher, car ils masquent la richesse et la diversité de chacun des deux groupes linguistiques. Il souligne : " Finally, I think we English Canadians must get to work on what I am inclined to call nationalizing, or, if you prefer, bi-culturalizing, the Dominion government and all its departments and emanations ${ }^{68}$. " Pour ce faire, il faut que les francophones se sentent plus confortables à Ottawa et qu'ils soient plus nombreux à être nommés à des postes de prestige. Le nationalisme en construction, s'il veut être porteur d'avenir, doit respecter le biculturalisme. Forsey conclut son allocution en mentionnant qu'il appuie la proposition d'enquête formulée par André Laurendeau. Il rappelle l'importance de faire honneur à la vision des Pères de la Confédération, qui reposait sur le partenariat

${ }^{68}$ Eugene A. Forsey, "Canada: Two Nations or One? ", The Canadian Journal of Economics and Political Science = Revue canadienne d'économique et de science politique, vol. 28, nº 4 (novembre 1962), p. 497. 
entre anglophones et francophones. Il faut maintenant donner une nouvelle impulsion à cette vision :

A Canadian Anglo-French partnership was the vision of Cartier and Macdonald. It is not obsolete. In the contemporary world of developing internationalism, it is even more necessary than in the nineteenth century world of triumphant nationalism. For Canadian nationalism can be not a dividing, but a uniting nationalism. I say "can be". The Fathers wrought well, and laid our foundations deep and strong. But the building is still unfinished, and parts of it have suffered from damage through the years. It does not need a bombing squad or a wrecking crew. But it does need alterations, repairs, additions, the expansion of certain rooms; and all of us have to be made to feel at home in it ${ }^{69}$.

Dans un article du Canadian Forum paru en octobre 1963, soit quelques mois à peine après l'annonce de la tenue de la commission Laurendeau-Dunton, l'historien Blair Neatby exhorte ses compatriotes anglophones à jeter un regard critique sur le passé pour ne pas répéter les erreurs commises : "English Canadians should admit that in the past they have only grudgingly accepted the existence of French Canada. On many occasions we have tried to impose policies which ignored or threatened its existence ${ }^{70}$. "L'article de Neatby témoigne donc d'un discours autocritique présent chez certains anglophones qui aspirent à un futur différent, qui sont prêts à s'engager pour que les francophones puissent être mieux reconnus à l'intérieur de la fédération canadienne. Neatby veut réfléchir au problème, mais surtout participer à la recherche de la solution. André Laurendeau publie aussi, dans The Canadian Forum, un article intitulé "The Search for (bicultural) Balance » quelques mois avant la mise sur pied de la Commission en avril 1963. À la fin des années 1950 et au début des années 1960, la revue Maclean's publie une série d'articles visant à faire découvrir le

69 Ibid., p. 501.

${ }^{70}$ Blair Neatby, "The Present Discontents: A Proposal ", The Canadian Forum (octobre 1963). 
Québec au Canada anglophone. Parmi ceux-ci, se trouvent, entre autres, les articles de Hugh MacLennan, "One Canada: The Real Promise of Quebec's Revolution ", d'Eric Hutton, "How to Win Friends and Really Learn French (or English) », et de Paul Gzowski, "What Young French Canadians have on their Minds " ${ }^{71}$.

\section{Une initiative plus personnelle : le dialogue entre une Canadienne anglaise et une Canadienne française}

Si les initiatives citées précédemment sont l'apanage d'hommes engagés, certaines femmes aspirent, toutefois, à faire entendre leur voix et à contribuer à leur manière au dialogue. Comme elles sont le plus souvent écartées des rencontres intellectuelles, c'est par des initiatives personnelles que se cristallise leur effort. En 1963, paraît Chers ennemis : une Canadienne anglaise et une Canadienne française se disent leurs quatre vérités, un échange épistolaire entre Solange Chaput-Rolland et Gwethalyn Graham. Dès la préface, les auteures soulignent l'aspect sain et porteur d'espoir du dialogue :

Le dialogue est une des préoccupations les plus grandes des sociétés démocratiques et il nous semblait qu'il pourrait émerger quelque chose de valable de nos conversations. Ces lettres, écrites à SaintJérôme, à Montréal et à Paris, nous ont permis de comprendre la portée de cette pensée de Jean Le Moyne : "Mon héritage français, je veux le conserver, mais je veux tout autant garder mon bien anglais et aller au bout de mon invention américaine. Il me faut tout ça pour faire l'homme total. "Si un jour les Canadiens anglais et les Canadiens français récoltent les richesses incommensurables que leur offre une culture située au carrefour de Molière, de Shakespeare et de Melville, ils réaliseront leur plénitude d'être. Ce livre désire simplement aider à supprimer le

${ }^{71}$ Hugh MacLennan, "One Canada: The Real Promise of Quebec's Revolution ", Maclean's, vol. 74 (août 1961), p. 15, 32-34; Eric Hutton, "How to Win Friends and Really Learn French (or English) ", Maclean's, vol. 71 (août 1958), p. 22-25, 32-34; et Paul Gzowski, "What Young French Canadians have on their Minds ", Maclean's, vol. 76 (avril 1963), p. 21-23, 39-40. 
rideau de préjugés qui en ce moment divise les Canadiens entre eux, entraînant ainsi un risque de suicide national ${ }^{72}$.

Les deux femmes se sont rencontrées lors d'une action commune menée par des francophones et des anglophones en décembre 1962 au cours de laquelle une escouade de "Canadiens de tous les milieux, représentant officiellement les deux groupes ethniques du Canada ${ }^{73}$ " s'étaient réunis au Parlement pour remettre un mémoire à Howard Green et convaincre les ministres de ne pas s'engager dans le nucléaire. C'est donc cette amitié récente entre deux femmes qui donne lieu à la publication de ce dialogue que représente Chers ennemis, ouvrage publié à la fois en anglais, chez MacMillan Company of Canada Limited, et en français, aux Éditions du Jour. Dès les premières pages du dialogue, Gwethalyn Graham souligne à quel point les rencontres entre les deux communautés culturelles principales se font rares et débordent du cadre de la bienséance :

Je ne peux m'expliquer mieux qu'en disant que vous êtes la première Canadienne française qui m’ait invitée chez elle depuis vingt ans. Oh! Je rencontre des Canadiens français de temps en temps, bien entendu, généralement dans des organisations anonymes ou chez des Européens récemment arrivés qui n'ont pas encore compris les règles qui gouvernent la vie sociale au Québec, fondées sur l'opposition de types humains abstraits. De toute évidence, on n’invite pas à dîner des abstractions... ${ }^{74}$

Ces rapprochements, ces tentatives de compréhension approfondie et savante des relations canado-québécoises, sont nés d'une prise de conscience de l'état stagnant des rapports entre anglophones et francophones à la veille des célébrations entourant le premier centenaire de la Confédération. Les deux voisins cohabitaient depuis

72 Solange Chaput-Rolland et Gwethalyn Graham, Chers ennemis : une Canadienne anglaise et une Canadienne française se disent leurs quatre vérités, Montréal, Éditions du Jour, 1963, p. 17.

73 Ibid., p. 16.

74 Ibid., p. 24. 
près d'un siècle et ne collaboraient pas autant qu'ils auraient pu le faire dans les milieux intellectuels, politiques ${ }^{75}$, économiques ${ }^{76}$ ou culturels.

\section{Dans les journaux : la série dans La Presse, dirigée par Jean- Louis Gagnon, et les articles d'André Laurendeau dans Le Devoir}

En 1959, Jean-Louis Gagnon, rédacteur en chef de La Presse, invite le jeune journaliste et écrivain torontois Scott Symons, à venir faire un stage d'un an à son journal afin d'étudier les relations entre les deux cultures ${ }^{77}$. Né en 1933, Symons fait partie de l'élite torontoise. Après des études en histoire au Trinity College, il poursuit son parcours académique en littérature anglaise à l'Université de Cambridge $^{78}$. Francophile, il arrive au Québec en 1958 après s'être fait renvoyé de son emploi au Telegram de Toronto en raison de son esprit rebelle. Il est alors engagé au Chronicle Telegraph et se met à l'apprentissage du français; il souhaite « explorer le côté français de ce qu'il considère comme son patrimoine canadien ${ }^{79}$ ». Il fait sensation dans les milieux francophones en raison de son ouverture à l'Autre; il insiste d'ailleurs pour parler en français. Il est reçu chez Maurice Duplessis, il se rend à un réveillon de Noël chez André Laurendeau et fréquente la haute société canadienne-française. Comme le relate la littéraire Susan Margaret Murphy, qui s'est

75 Sur la scène fédérale, les ministères de prestige, les postes de hauts conseillers demeuraient, sauf quelques exceptions, l'apanage des anglophones.

${ }^{76}$ La sous-représentation des francophones dans les hautes sphères de décision des milieux économiques était une intuition qu'avaient plusieurs au début des années 1960, intuition qui deviendra une réalité pour certains lorsque les études de la commission Laurendeau-Dunton présenteront des chiffres. Voir, notamment, les études de l'économiste André Raynaud soumises à la Commission : " La propriété des entreprises au Québec " et "La répartition des revenus entre les groupes ethniques ». Cette dernière étude est cosignée par G. Marion et R. Béland.

77 Murphy, Le Canada anglais de Jacques Ferron, p. 86.

78 Ibid., p. 76.

79 Ibid., p. 77. 
intéressée au passage de Symons au Québec : «Avec le mandat large accordé par Jean-Louis Gagnon, de pair avec son caractère fort, ses positions controversées, son intelligence, sa sensibilité, sa ténacité et sa maîtrise du français, Symons a su faire la connaissance de personnes influentes dans divers milieux canadiens-français ${ }^{80}$. " Personnage singulier et flamboyant, admiré de certains et dédaigné par d'autres ${ }^{81}$, il poussait même son amour de la composante francophone du pays jusqu'à se dire nationaliste canadien-français ${ }^{82}$.

Le stage de Symons comme chroniqueur à La Presse l'amène à publier une série d'articles entre le 25 mars et le 10 avril 1961, dans une rubrique intitulée "Le Canada: duel ou dialogue ». Il résume ainsi son expérience couronnée de succès au quotidien montréalais :

Il y a un an presque, je faisais la route vers Saint Sauveur des Monts [...]. Je venais d'arriver de Paris, pour m'installer pendant une année pour reporter à "La Presse ", à l'invitation de JeanLouis Gagnon. Son idée, ainsi que la mienne, c'était d'exposer un jeune Torontois aux problèmes et aux espoirs du Canada français. Personne à La Presse, moi-même surtout, n’avait une idée précise de ce que je devais faire pour le journal et certes personne ne prévoyait une série d'articles qui aurait un succès fou $[\ldots]^{83}$.

La série de Symons récolte non seulement un succès populaire, mais également un succès critique en méritant un des premiers prix du Cercle des journalistes de Montréal (Montreal Men's Press Club) ${ }^{84}$. S’il peut sembler singulier qu'un anglophone s'intéresse autant à ses homologues francophones, allant jusqu'à soutenir les causes de ces derniers, il n'en reste pas moins que Symons était un homme de son

80 Ibid., p. 86.

81 Jacques Godbout et Jacques Ferron ne faisaient pas nécessairement partie de son cercle d'admirateurs. Pour Ferron, il semblait suspect qu'un anglophone s'intéresse autant aux francophones. Godbout, quant à lui, trouvait Symons arrogant et provocateur (Ibid., p. 87).

82 Entretien avec Naïm Kattan du 7 mai 2007, dans Ibid., p. 87.

${ }^{83}$ Scott Symons, "Ici Toronto : un volontaire pour St-Sauveur-des-Monts ", Le Nouveau Journal, 15 octobre 1961, p. 4, cité dans Ibid., p. 87-88.

${ }^{84}$ Ibid., p. 78. 
temps; comme d'autres autour de lui, il souhaitait un rapprochement entre les deux peuples fondateurs. En allant à la rencontre de l'Autre, en s'intéressant à lui et en lui consacrant des articles, il aspire à détruire ce mur qui sépare les "deux solitudes". Il reprend des thèmes récurrents à l'époque, notamment la menace américaine, et exhorte les Canadiens anglophones à aller à la rencontre des francophones et les deux groupes principaux à collaborer davantage $e^{85}$.

Plus que tous les éléments cités auparavant, une série d'articles influence directement la mise sur pied de la commission LaurendeauDunton. Il s'agit d'articles publiés par André Laurendeau au début des années 1960 dans Le Devoir. Sensible aux changements qui s'opèrent au Québec et dans le reste du pays, l'éditorialiste du Devoir appelle à la création d'une enquête pour étudier les questions liées au bilinguisme et au biculturalisme. Au départ, c'est le piètre état du bilinguisme qui l'interpelle et le pousse à prendre la plume. En janvier 1962, André Laurendeau publie l'éditorial considéré comme le déclencheur de la Commission royale d'enquête sur le bilinguisme et le biculturalisme. Le 26 janvier, l'éditorialiste du Devoir signifie son mécontentement envers les chèques bilingues du gouvernement fédéral. À ses yeux, c'est trop peu, trop tard. Il faut entreprendre une vaste enquête sur l'état du bilinguisme : «Le bilinguisme des chèques, c'est une mesure tardive qui ne répond aucunement aux aspirations actuelles des Canadiens français. Ils en ont assez de ces concessions à la petite décennie. Ils demandent, si l'on tient à leur présence au sein de la Confédération, une réforme autrement générale ${ }^{86}$. "

Cet éditorial eut un retentissement sur la scène politique; Lester B. Pearson, alors chef de l'opposition, intègre le projet d'enquête demandé par l'éditorialiste du Devoir dans son programme électoral présenté en vue de l'élection de juillet $1963^{87}$.

85 Voir Scott Symons, "L'Américanisme : ennemi commun et héritage aigre-doux ", La Presse, 5 avril 1961, p. 5.

86 André Laurendeau, "Pour une enquête sur le bilinguisme », Le Devoir, 20 janvier 1962, p. 4.

87 Ramsay Cook, "Le Canada vaut une enquête ", International Journal, vol. 23, n 2 (printemps 1968), p. 291. 
$\mathrm{Si}$, au départ, l'enquête souhaitée ne touchait qu'à la question du bilinguisme, avec le temps, le projet se précise dans l'esprit de son créateur. En mars 1962, André Laurendeau revient à la charge et brosse avec davantage de précision les contours de l'étude désirée portant sur le Canada, une étude unissant anglophones et francophones :

Je ne demande qu'une étude honnête de la question - et notamment une enquête systématique sur la façon dont les États où le problème se pose l'ont résolu (par exemple, la Suisse et la Belgique); une autre enquête, aussi poussée que possible, sur les motifs qui empêchent à l'heure actuelle les Canadiens français de participer autant qu'ils le devraient et le pourraient à l'administration de leur pays.

On me fera remarquer que ces suggestions ne tiennent aucun compte des frontières constitutionnelles : c'est exact. J'examine en ce moment ce que devrait être un État bilingue : or, dans une Confédération, ses pouvoirs sont morcelés. Il faudrait donc que les réformes proposées reçoivent l'adhésion de onze gouvernements. N'est-ce pas signaler au départ qu'elles sont parfaitement irréalisables?

À l'heure actuelle, en tout cas, je ne sache pas que l'État centre ou qu'un seul des États provinciaux soit prêt à en accepter l'idée. L'expérience pourrait être tentée d'une autre manière.

Existe-t-il au Canada anglais, des individus assez nombreux et assez éclairés pour accepter d'examiner cette " utopie »? J'imagine qu'ils se réunissent, qu'ils forment, avec des Canadiens français, une sorte de constituante officieuse, qu'enfin ils arrivent à se mettre d'accord sur un programme commun. Autour du programme ainsi dégagé, une campagne d'opinion est amorcée dans toutes les parties du Canada.

A-t-elle des chances de succès? Il y a quelques années, je n'aurais même pas osé poser la question. Mais deux faits, il me semble, rendent cette expérience imaginable : d'une part, la réaction d'un certain nombre d'Anglo-Canadiens devant l'américanisation de notre pays : d'autre part, le séparatisme québécois. Ces deux 
facteurs nouveaux ont assurément modifié les vues de plusieurs Anglo-Canadiens. Il s'agirait de voir jusqu'où ${ }^{88}$.

Cet extrait de l'éditorial d'André Laurendeau résume bien l'esprit du début des années 1960. Les temps ont changé, et il est possible de croire, en 1962, à une collaboration fructueuse entre anglophones et francophones pour penser ensemble les voies de l'avenir. Des éléments font également en sorte qu'il y a urgence d'agir. La montée du souverainisme revendicateur, avec la création du Rassemblement pour l'indépendance nationale (RIN), et la volonté de trouver une solution à la montée en force de l'influence culturelle et économique des États-Unis, poussent les intellectuels et les responsables politiques à agir. Une des solutions apparaissant dans ce contexte est de repenser le Canada pour que sa dualité s'exprime davantage et ainsi renforcer le caractère spécifique du pays face aux États-Unis, tout en répondant aux revendications autonomistes du Québec en rééquilibrant les pouvoirs.

\section{Le réseau s'articulant autour de la commission Laurendeau-Dunton}

À travers le récit de toutes ces tentatives pour susciter des échanges entre les deux principaux groupes culturels du Canada se dégage un certain modèle d'engagement autour duquel se rassemble un réseau d'intellectuels, d'universitaires et de responsables politiques pour penser autrement le Canada et réfléchir à l'épanouissement de sa dualité. S’il est faux de penser que toute la communauté intellectuelle de l'époque se positionne en faveur d'un rapprochement entre les deux solitudes, il est tout de même possible de constater que les années de l'après-guerre ont vu se constituer un réseau d'hommes et de femmes s'unissant autour des thématiques du bilinguisme et du biculturalisme. Aux yeux de ces hommes et de ces femmes, la réforme

${ }^{88}$ André Laurendeau, Ces choses qui nous arrivent: chronique des années 19611966, Montréal, Éditions HMH, 1970, p. 62-63. 
du pays passait par l'intensification du dialogue et une meilleure reconnaissance de la dualité du Canada. Les avenues explorées pendant les années précédant la commission Laurendeau-Dunton sont multiples; elles constituent des exemples à petite échelle de ce que deviendra la Commission, qui incarne le plus grand laboratoire intellectuel consacré à l'étude du Canada jamais mis sur pied à l'époque $^{89}$. De la création d'ouvrages bilingues à l'organisation de congrès réunissant des anglophones et des francophones désireux de penser la dualité; de la conception de revues à la publication d'articles lançant un cri d'alerte dans les journaux, les initiatives témoignent de créativité, mais surtout du climat qui règne à l'aube de la Commission. Un climat d'inquiétude, certes, mais aussi un climat d'espoir face à l'avenir et d'éclosion de nouvelles idées pour penser cet avenir sous le signe de la réconciliation.

Afin de mieux comprendre la commission Laurendeau-Dunton, il est donc pertinent de présenter les démarches consacrées à des thématiques similaires entreprises dans les années la précédant. Ces démarches sont éclairantes, car elles permettent de constater que les thématiques reprises dans le mandat de la Commission constituent des enjeux phares au cours des années qui la précèdent. Elles mettent également en lumière la constitution d'un réseau intellectuel qui a contribué à la création de la Commission et soutenu son déploiement. Comme le rappellent Stéphanie Angers et Gérard Fabre, « la notion de réseau intellectuel permet d'articuler savoirs et pratiques, ces deux registres étant forcément imbriqués : les intellectuels ne vivent pas dans des monades, ils doivent faire face à des enjeux collectifs à partir desquels ils se définissent et produisent des connaissances ${ }^{90}$ ". Dans les années 1950 et 1960, le bilinguisme et le biculturalisme, deux

89 Michael Oliver, "Laurendeau et Trudeau : leurs opinions sur le Canada ", dans Hudon et Pelletier (dir.), L'engagement intellectuel, p. 339.

90 Stéphanie Angers et Gérard Fabre, Échanges intellectuels entre la France et le Québec, 1930-2000 : les réseaux de la revue Esprit avec La Relève, Cité libre, Parti pris et Possibles, préface de Marcel Fournier, Québec, Les Presses de l'Université Laval, 2004, p. 3. 
concepts à la base du mandat de la Commission, représentent des enjeux autour desquels se rassemblent les intellectuels. La notion de dialogue et la volonté d'intensifier la collaboration entre les deux peuples fondateurs sont également très présentes dans les milieux intellectuels. Ce n'est pas un hasard si, parmi les personnalités présentées précédemment, plusieurs occupent des postes clés à la Commission. Ces intellectuels, de par la multiplication de leurs questionnements et la création de tribunes pour les exprimer, ont créé un terrain propice à l'émergence d'une vaste enquête. Si la Commission a pu prendre son envol en 1963, c'est parce qu'il y avait non seulement un terrain pour l'accueillir, mais surtout des gens prêts à lui donner vie, car ils avaient déjà entamé la réflexion.

André Laurendeau a participé au Congrès des affaires canadiennes ${ }^{91}$, en plus de publier dans Exchange et d'écrire la série d'articles dans Le Devoir demandant la mise sur pied d'une commission d'enquête se consacrant au bilinguisme et au biculturalisme. Il apparaît donc naturel de faire appel à lui, par la suite, pour présider la Commission. Jean-Louis Gagnon, instigateur de la série d'articles dans $\mathrm{La}$ Presse intitulée " Le Canada : duel ou dialogue », devient commissaire. Frank Scott, qui a participé au projet du Comité pour les deux cultures rejoint également les rangs de la Commission à titre de commissaire. Michael Oliver collabore au Congrès des affaires canadiennes avant d'être nommé directeur de la recherche de la Commission. Celui qui deviendra le conseiller spécial à la recherche de la Commission, Léon Dion, publie dans Exchange en 1961. De plus, il joint sa voix à celle d'André Laurendeau pour demander, lui aussi, la tenue d'une commission d'enquête fédérale. Le 8 décembre 1962, il publie un article dans Le Devoir où il " démontre l'insignifiance de la participation des Canadiens français à la direction des entreprises du secteur privé, au Québec même et dans la fonction publique fédérale ${ }^{92}$ ». Plusieurs

${ }^{91}$ En 1961, il publie "Le Canada, une nation ou deux? ", dans Association des étudiants de l'Université Laval, Le Canada, expérience ratée... ou réussie?, p. 71-87.

92 Dion, La révolution déroutée, p. 194. 
universitaires vont poursuivre et approfondir leur réflexion pendant la tenue de la Commission. Les échanges entamés dans les années précédant la Commission entre anglophones et francophones s'intensifient au cours de ses travaux. Les initiatives mises sur pied à partir des années de l'après-guerre ont tenté de faire en sorte que les Canadiens anglophones et francophones cessent de coexister pour commencer à collaborer. En suscitant des rencontres entre anglophones et francophones, en aspirant à refléter la dualité canadienne et à respecter le bilinguisme, elles ont mis la table pour ce qui allait suivre. La Commission représente, en quelque sorte, l'aboutissement de ces efforts. Le réseau intellectuel qui a commencé à se tisser au cours des années 1940 autour d'une volonté de dialoguer s'est rassemblé, au départ, en raison de certaines inquiétudes communes : la peur de l'annexion aux États-Unis et la crainte de voir éclater le pays devant la montée du souverainisme au Québec. Il s'est structuré autour d'une volonté d'agir avant qu'il ne soit trop tard. Il a mis en scène des hommes et des femmes dont l'engagement envers le pays les a poussés à se questionner sur les meilleures options à envisager pour l'avenir, en réfléchissant aux thématiques que sont l'unité canadienne, la survie du Canada et la dualité. Leur engagement et leurs activités traduisent une sensibilité exacerbée pour ces questions.

Il semble également que la curiosité intellectuelle a créé une émulation dans l'espace politique, transmettant, au passage, ce désir d'ouverture à l'Autre aux responsables politiques. Lester B. Pearson fit d'ailleurs un discours à la Chambre des communes en décembre 1962 afin de solliciter l'appui des députés et du premier ministre Diefenbaker dans la mise sur pied d'une enquête sur le bilinguisme et le biculturalisme. Sa requête étant demeurée lettre morte, il écrivit une lettre à Diefenbaker en mai 1962 pour lui rappeler la nécessité d'une telle enquête :

I suggested that a broad and comprehensive inquiry should be conducted, in consultation with the provinces, on bilingualism and biculturalism. That proposal received widespread support in Parliament, I believe, in the country. 
I am now writing to ask whether your government would favour such an inquiry by a Royal Commission [... $]^{93}$.

Diefenbaker était complètement fermé à l'idée d'une telle enquête. Selon l'historien Jack L. Granatstein, son gouvernement ne parvenait pas à comprendre ce qui se passait au Québec. Plutôt que d'apaiser le climat, son attitude ne contribuait qu'à la dégradation des rapports entre les francophones et les anglophones: "The battle between French and English Canada was heating up, made fiercer by the Diefenbaker government's total inability to comprehend what was taking place in Québec ${ }^{94}$. " Le premier ministre conservateur défendait la conception d' " un Canada, une nation ». Il apparaissait donc improbable que ce « défenseur de l'idéal d'une nationalité canadienne sans trait d'union ${ }^{95}$ " donne vie à l'ébauche de l'enquête pensée par André Laurendeau, Lester B. Pearson et les autres intellectuels qui avaient voulu étudier en profondeur les relations entre le Canada français et le Canada anglais. Au moment où Diefenbaker perd son élection au profit de Lester B. Pearson, qui prend la tête d'un gouvernement libéral minoritaire le 8 avril $1963^{96}$, le terrain apparaît

${ }^{93}$ Lettre de Lester B. Pearson au premier ministre Diefenbaker, mai 1962, Bibliothèque et Archives Canada, Fonds Jaroslav-Rudnyckyj, MG31-D58, vol. 5.

94 J. L. Granatstein, Canada 1957-1967: The Years of Uncertainty and Innovation, Toronto, McClelland and Stewart, 1986, p. 247-248. L'incompréhension patente de John Diefenbaker envers le Québec est d'ailleurs soulignée à quelques endroits dans la littérature scientifique. Voir Peter Regenstreif, The Diefenbaker Interlude: Parties and Voting in Canada: An Interpretation, Toronto, Longmans Canada, 1965, p. 109-132. Voir également Kenneth McRoberts, Un pays à refaire : l'échec des politiques constitutionnelles, Montréal, Éditions du Boréal, 1999.

95 McRoberts, Un pays à refaire, p. 75.

${ }^{96}$ Les libéraux se retrouvent alors avec 129 sièges, les progressistes conservateurs avec 95 et les créditistes avec 20 sièges. C'est au Québec que l'élection s'est jouée. Après avoir perdu plusieurs sièges aux dernières élections de 1962, les libéraux retrouvent les $46 \%$ de voix qu'ils avaient déjà obtenues au temps de Louis Saint-Laurent. Le manque de sensibilité de John Diefenbaker envers les enjeux québécois a sans doute, en partie, donné ce résultat (André Bernard, "Vingt ans de pouvoir libéral à Ottawa : la conjoncture politique ", dans Yves Bélanger et Dorval Brunelle (dir.), L’ère des libéraux : le pouvoir fédéral de 1963 à 1984, Québec, Presses de l'Université du Québec, 1988, p. 16-17). 
plus propice que jamais à la concrétisation du souhait d'André Laurendeau. Le diplomate de carrière croyait en la possibilité d'enrichir le dialogue et aux vertus d'une enquête. C'est ainsi que la Commission royale d'enquête sur le bilinguisme et le biculturalisme fut créée en juillet 1963. En réunissant plus de 400 universitaires de partout à travers le pays pour étudier les enjeux liés au mandat, en créant des espaces de dialogue d'un océan à l'autre, en demandant aux commissaires de penser leur Canada idéal, la Commission a défriché les pistes ouvertes dans les années qui l'ont précédée.

\section{Conclusion}

Le fil conducteur d'un récit se dégage des études et des réflexions menées au cours des décennies qui ont précédé la Commission, le récit de la fondation, pierre par pierre, contribution par contribution, de la commission Laurendeau-Dunton. Des premiers balbutiements de l'étude de la dualité du Comité sur l'étude des deux cultures, en passant par les Congrès des affaires canadiennes, par la venue de Scott Symons au Québec et sa série d'articles consacrée à la cohabitation des deux peuples fondateurs, les premiers jalons de la Commission peuvent être observés. Ces efforts, s'ils n’ont pas tous récolté un succès éclatant, ont tout de même fait ressortir cette volonté de dialogue et cet intérêt pour les deux notions clés de la Commission : le bilinguisme et le biculturalisme. La Commission n'a donc pas surgi de nulle part. On reproche parfois aux commissions d'enquête de représenter des créatures factices, imaginées par les responsables politiques pour masquer d'autres problèmes. Ce n'est pas le cas de la commission Laurendeau-Dunton. Celle-ci s'inscrit dans un processus de nation-building, alors que le pays, après presque un siècle d'existence, se trouve à la croisée des chemins. Entre la peur de l'annexion aux États-Unis et la volonté d'éviter l'éclatement du pays en raison de l'affirmation d'un souverainisme revendicateur au Québec, les initiatives ayant précédé la Commission ont voulu trouver une voie spécifiquement canadienne et ouvrir le chemin vers 
la réconciliation entre les deux peuples fondateurs en suscitant des rencontres et des débats. Les années 1960 ont frappé l'imaginaire en raison des courants plus radicaux qui les ont marquées. Toutefois, à côté de la montée du souverainisme ou, encore, de la fermeture de certains Canadiens anglophones aux demandes des francophones, les années 1960 demeurent une décennie fascinante pour l'étude des rapprochements entre les « deux solitudes ». Les années qui ont suivi la Commission, au cours desquelles le multiculturalisme est devenu plus populaire que le biculturalisme, font souvent oublier toute cette énergie déployée dans les années 1960 pour donner un élan biculturel au Canada, pour créer un dialogue durable. 